The theory posited unknown and perhaps inaccessible degrees of freedom of quantum systems, the values of which fix the behaviour of such systems. But quantum indeterminism is overcome at the price of allowing 'non-local' or 'faster-than-light' communication among those hidden variables, which might conflict with Einstein's theory of relativity. Gilder devotes long discussions to the career and ideas of Bohm. All the more puzzling, then, that when she tells us again and again about experiments confirming violations of the Bell inequality, she leaves the reader with the impression that this is a handsdown victory for quantum mechanics over hidden variable theories, when it is only local hidden variable theories that are put in jeopardy. One might reject Bohmian mechanics for other reasons, but these tests alone do not afford them. Kumar's hasty resumé of the same results leaves one still more confused. He suggests that Einstein's reservations about the completeness of quantum mechanics are somehow vindicated by experiments confirming the predictions of standard quantum mechanics.

Both authors would have been helped by a closer look at the well-known re-analysis of Bell's argument, introduced in 1984 by Jon Jarrett and refined by Shimony. Jarrett showed that the original Bell locality condition is a conjunction of two logically independent conditions that Shimony called outcome independence and parameter independence. The former is akin to a denial of quantum entanglement, the latter to relativistic locality constraints. Experimental violations of the Bell inequality can now be traced to violations of one or the other of these two conditions. That there are two independent routes to violations of the Bell inequality makes it clear how orthodox quantum mechanics, which presumes entanglement, and a Bohm-type hidden variables theory, which assumes relativistic non-locality at the microlevel, can both claim to have been vindicated by the Bell experiments. But these gentle criticisms should not deter the interested reader from enjoying two welcome additions to the popular history of twentieth-century physics.

Don Howard is professor of philosophy at the University of Notre Dame, Notre Dame, Indiana 46556, USA. His book on Einstein for Blackwell's Great Minds series will be published in 2009. e-mail:dhoward1@nd.edu

\title{
Back to the roots of crop farming
}

\section{Where Our Food Comes From: Retracing Nikolay Vavilov's Quest to End Famine by Gary Paul Nabhan \\ Island Press: 2008.266 pp. \$24.95, £21.50}

In 1941, when German and Finnish troops threatened to besiege the Russian city of Leningrad (now St Petersburg), Soviet leaders hurried to authorize the evacuation of the art collection from the city's Hermitage museum. Another extraordinary treasure, then the world's largest collection of more than 380,000 food crop samples housed at Leningrad's All-Union Institute of Agricultural Sciences, did not receive such privileged treatment; it survived the 1941-44 Leningrad blockade only through the virtue of committed individuals. Clearly, society places different values on the heritage content of the art museum and the seed bank.

In Where Our Food Comes From, Gary Paul Nabhan, a conservationist and research social scientist at the University of Arizona, spotlights crop diversity as a neglected but vital cultural resource. He does so by chronicling the journeys of Nikolay Vavilov, Russia's famous geneticist and botanist, and creator of the Leningrad seed bank. Vavilov was the first to identify the world's centres of crop diversity - a concept that, decades later, was developed by conservation biologists into what we now call biodiversity hotspots or conservation targets. Based on narratives from Vavilov's expeditions in the 1920s and 1930s and the author's own travels to retrace Vavilov's steps, Nabhan looks at changes in agricultural biodiversity.

The journeys cover nine regions of the globe. Each chapter highlights a specific land-use system, such as the wild apple forests of Kazakhstan; the diverse date cultivation in the oases of the Maghreb in north Africa; the traditional milpa cropping system in Mexico, based on maize (corn) and beans; or the forest gardens of

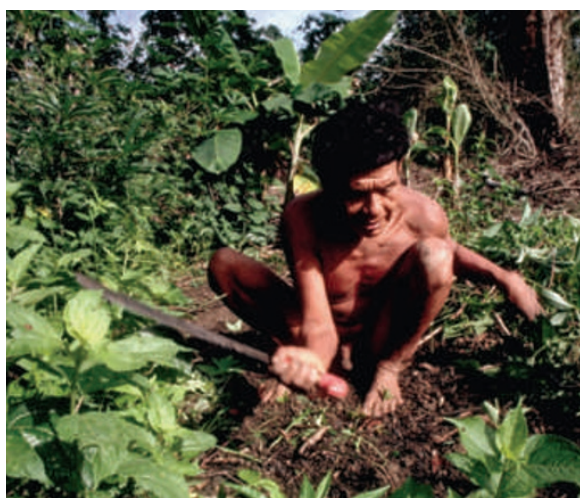

Amazon agriculture benefits from crop diversity. the Amazon basin in South America. Nabhan observes that, almost everywhere, crop diversity has strongly and rapidly declined during the time between Vavilov's and his own visits. Both global- and regional-scale processes are held responsible for the homogenization of crops. For example, melting glaciers account for a depletion of hydrological sources in Tajikistan's Pamir mountains, which damages traditional cropping systems. In northern Italy's Po valley, wasteful irrigation practices and mechanized high-performance agriculture have displaced traditional land uses. In the Middle East, political unrest and the legacy of colonialism have disrupted ancient farming systems. In Kazakhstan, economic growth has driven urban sprawl, threatening agricultural land close to cities.

Yet Nabhan's perspective is positive. He stresses the value of diverse crop varieties and the cultural traditions of land use and food preparation - for pragmatic reasons and as a cultural heritage that provides humankind with pleasure. He presents promising initiatives to recover crop diversity, from the rehabilitation of farmers' markets in Lebanon to efforts to renew local Hopi food systems in Arizona. And he shows how rich crop diversity and traditional seed selection and distribution support adaptation to changing environmental or societal conditions. I was fascinated by the story of how Colombian peasants have selected varieties of coca plants - the leaves of which contain cocaine alkaloids - that are resistant to the aerially sprayed herbicide used in the war on drugs. These resistant varieties have arisen through farmer-based selection, adaptation and informal dissemination of coca cuttings, all without any genetic engineering.

Where Our Food Comes From is a marked critique of the worldwide simplification of agricultural systems. It pins its hopes on local, traditional agriculture and is sceptical of topdown approaches to increasing food production, such as calls for another 'green revolution'. Many of its conclusions coincide with those reached by the International Assessment of Agricultural Knowledge, Science and Technology for Development, which concluded that the focus on maximum agricultural commodity production was responsible for the depletion of natural capital.

There are two opposing sides in the debate on how to increase food security. One side, which includes Nabhan, endorses the support of small-scale, low-output agriculture that is highly diverse. Among other examples, Nabhan uses the case of Ethiopian wheat varieties, which proved invaluable in the fight against 
the virulent leaf rust fungus, to show that traditional, allegedly outmoded forms of land use may help to solve modern environmental problems. The opposing side advocates modern, technology-intensive, high-performance forms of agriculture that safeguard large parts of the world's food supply today, but are not resilient and depend on significant inputs of fossil fuels that may become expensive in the future.

Nabhan notes the often overlooked contributions of traditional ecological knowledge to sustainable food production. After reading the book, major questions arise. Do locally domesticated plants have an archival function that provides genetic varieties for modern plant breeding, and should they thus be conserved in their remote centres of origin? Or can they be used on a larger scale in intensive agricultural landscapes? The integration of traditional and modern practices into agricultural systems that are productive yet sustainable, consideration of the needs of small-scale farmers and maintenance of diverse ecosystems will all remain conflict-laden - but they represent crucial challenges in the quest to feed the world. Tobias Plieninger heads a group on ecosystem services at the Berlin-Brandenburg Academy of Sciences and Humanities, Jägerstrasse 22/23, 10117 Berlin, Germany. e-mail: plieninger@bbaw.de

\section{Early days of science broadcasting}

\section{Science on the Air: Popularizers and Personalities on Radio and Early Television by Marcel Chotkowski LaFollette Chicago Univ. Press: 2008. 324 pp. $\$ 27.50$}

Writing popular science and writing about popular science are very different endeavours. The former is at least as old as John Newbery's Newtonian System of Philosophy (1761), with its star Tom Telescope, and has been practised in every medium in turn: lectures, books, newspapers, magazines, cinema, radio, television and the Internet. But analysing it is a recent pursuit, tied to the growth of university sciencestudies departments concerned with science's social dimensions. Although now established, the field is not so overpopulated that there is agreement about how to do it, or for whom.

Marcel Chotkowski LaFollette has written on the history of popular science for more than 25 years, and is best known for Making Science our Own: Public Images of Science, 1910-1955 (Univ. Chicago Press, 1990). In her new book, Science on the Air, she reveals from primary sources the story of the first 20 years of science broadcasting on US radio. We meet the Smithsonian Institution's Austin Hobart Clark, who initiated a series of scientists' radio lectures in 1923, and Thornton Burgess, who transferred from writing children's nature books to radio programmes. LaFollette discusses how the scientist William Ritter and the newspaper

publisher Edward Scripps established the Science Service news bureau in 1920, which was highly influential in both print and radio for four decades. The one post-war chapter, which benefits from an established secondary literature, discusses science on US television in the 1940s and 1950s. Here LaFollette con-
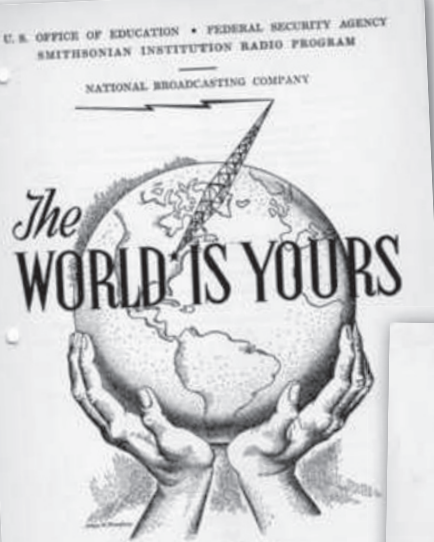

March of Science

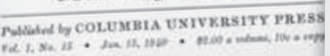
trasts the sober style of The Johns Hopkins Science Review (from 1948) with the more entertaining formats sponsored by the Bell Telephone System and programmes made by Disney from 1954.

argumentative or analytical approach would have addressed why popular science matters.

The relationship of science to people and to the broader culture was, in the period she describes, gaining importance as the sciences began to have a significant impact on ordinary lives. The possibility that scientists might have a negative impact, through their perceived responsibility for new weapons and mass-production machinery, was a live issue. More historical studies of the vehicles of science communication are needed so that we can understand the interplay between science and society; but this requires more than narrative.

So little is published in this area that it would be unfair to ask LaFollette to draw international comparisons. With a myriad of local commercial stations to follow and many potential stories to tell, she has a more difficult job than scholars of British science radio, for example, where for the same period there was one monopoly broadcaster, the BBC. LaFollette describes how in the United States scientists preferred a lecture format, but were put under pressure to make their programmes more entertaining or risk marginalization. In Britain, producers remained deferential and accepted that information should be conveyed directly from the authority to the viewer "public men personally discussing events in the news", as one producer put it. Unlike in the United States, elite scientific organizations such as the Royal Society and the British Association repeatedly sought control over broadcasts. A common element was the growing importance of the skilled intermediary, and mediation by a presenter was the norm on television by the late 1950 s.

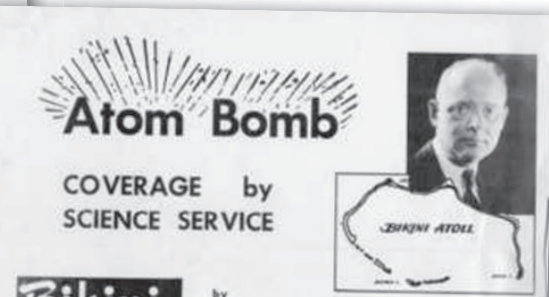

Bikini De mang BHONt Retogiv

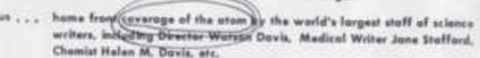

Scientists conveyed information directly to the public in early radio broadcasts.

Science on the Air has an academic level of detail. But LaFollette writes in a narrative style, with occasional touches of whimsy "microphones trembled during atomic bomb tests and rattled with impassioned debate", for example. This choice has consequences; after reading it, you will know what happened to US science radio and early television, but you may wonder why you need to know it. A more
Today the media market is global. Producers work in a multichannel world, where no country's output can afford to limit itself to national concerns. Single broadcasters can rarely afford the cost of ambitious science shows and so seek to collaborate. Co-production has led to a homogenization of style, moving closer to the US entertainment model and away from the earnest, deferential treatment LaFollette describes in 1920s radio. Some commentators fear that the science in science programming is reaching homeopathic concentration. The continuing presence of science in the schedules, evidently vulnerable to broadcasting fashions, demands investigation. This book shows it can be done.

Tim Boon is chief curator at the Science Museum, London, and author of Films of Fact: A History of Science in Documentary Films and Television. 